\title{
Erratum: Parity-time symmetry breaking in magnetic systems \\ [Phys. Rev. B 94, 020408(R) (2016)]
}

\author{
Alexey Galda and Valerii M. Vinokur
}

(Received 22 October 2019; published 8 November 2019)

DOI: 10.1103/PhysRevB.100.209902

We report an error in Fig. 2 of the original paper. The corrected version of Fig. 2 is shown below (Fig. 1 in this Erratum). Also, the original paper refers to three separate $\mathcal{P} \mathcal{T}$ symmetry-breaking transitions (at $\beta \approx 4.5,9.3$, and 9.8) for the Hamiltonian (7) with $h_{x}=1$ and $D=20$ on p. 2 of our paper. In fact, there exists only one such phase transition at $\beta=\beta_{1} \approx 4.5$, which corresponds to the Hopf bifurcation in the spectrum of the Hamiltonian. The other two points with coalescing eigenvalues correspond to homoclinic bifurcations and do not result in the appearance of the nonzero imaginary part in the spectrum, see Fig. 1(b).

Additionally, the characteristic time of magnetization dynamics for the material parameters used in the micromagnetic simulations was reported as $\tau \approx 0.14 \mathrm{~ns}$. The correct value is a factor of $2 \pi$ bigger, $\tau=2 \pi\left(\gamma H_{0}\right)^{-1} \approx 0.89 \mathrm{~ns}$.

The results and main conclusions of the paper remain unchanged.
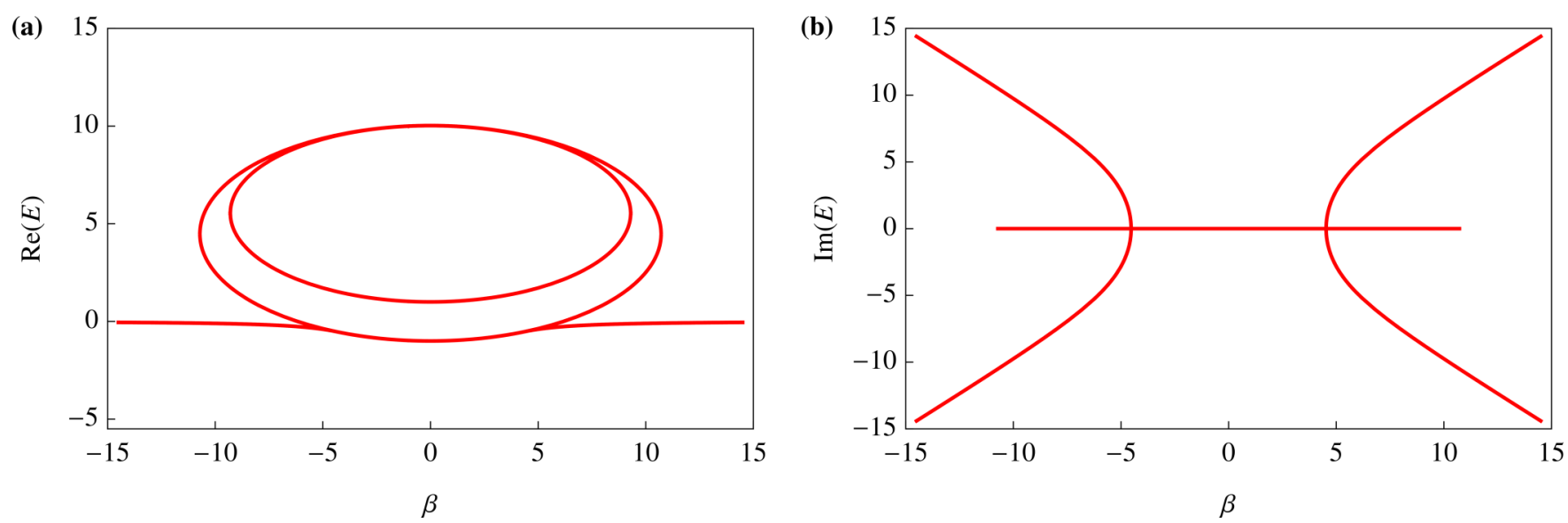

FIG. 1. (a) Real and (b) imaginary parts of energy spectrum of the Hamiltonian (7) from the original paper as a function of the spin-transfer torque parameter $\beta$ with $h_{x}=1$ and $D=20$. The $\mathcal{P} \mathcal{T}$ symmetry-breaking transition occurs at $|\beta|=\beta_{1} \approx 4.5$. 